\title{
Computational Investigation of Arc Behavior in an Auto-Expansion Circuit Breaker with Gases of $\mathrm{SF}_{6}$ and $\mathrm{N}_{2} * *$
}

\author{
Jinling Zhang
}

\begin{abstract}
An electric arc behavior in a full-scale $245 \mathrm{kV}$ autoexpansion circuit breaker with a moving contact has been carried out for two filling materials, $\mathrm{SF}_{6}$ and $\mathrm{N}_{2}$. The computer simulation involves the simultaneous solution of the three conservation equations (mass, momentum and energy) and Maxwell's equations. The Lorentz force due to the interaction between the arcing current and its own magnetic field, radiation model and Prandtl mixing length turbulence model have been taken into account. The features of temperature during the whole arcing period are obtained. Results show that the arc column does not shrink properly in radial direction with $\mathrm{N}_{2}$ filling.
\end{abstract}

Keywords-Computational simulation, arc behavior, autoexpansion circuit breakers, $\mathrm{SF}_{6}$-free gas

\section{INTRODUCTION}

$\mathrm{C}$ IRCUIT breakers are used in the transmission and distribution network to prevent damage to generators, transformers, cables and end user equipment from fault currents induced by accidents, or to satisfy the requirement of switching on/off. The breakers are designed to stop the current flow and to withstand voltage transients which arise on the electrical network when the current is interrupted. High-voltage circuit breakers are exclusively filled with Sulphur Hexaflouride $\left(\mathrm{SF}_{6}\right)$ since it is an excellence medium for extinguish the arc and has excellent insulation properties to withstand the induced voltage transients after arc extinction. However, the gas $\mathrm{SF}_{6}$ is a very strong greenhouse gas which has potency 22,800 times more than that Carbon Dioxide [1] and has a life time of about 4,000 years in the upper atmosphere. There is no other technology that is available to replace these circuit breakers. Therefore the replacement of $\mathrm{SF}_{6}$ with a more environmentally friendly gas is becoming an increasingly interesting research topic [2] and practically important issue for power equipment manufacturers and network operators [3]. Due to the complexity of the arc process in the circuit breakers, the

Jinling Zhang is with the Department of Electrical and Electronic Engineering, Xi'an Jiaotong Liverpool University, 215123, Suzhou, China.

**This project is supported by the Centre for Smart Grid and Information Convergence of Xi'an Jiaotong-Liverpool University, Suzhou, China performance of the circuit breakers depends on a number of design parameters and the whole arcing history. It is difficult or the cost would be intolerably high if the investigation solely depends on short circuit tests. It is therefore highly desirable to develop computer program to aid the circuit research and design, which would at least reduce the number of short circuit tests required.

An arc modelling based on the basic physical processes occurring in a circuit-breaker arc has been carried out for modern circuit breakers. In general, computer simulation of a modern circuit breaker arc involves the simultaneous solution of the three conservation equations (mass, momentum and energy) and Maxwell's equations with moving boundaries. The Lorentz force due to the interaction between the arcing current and its own magnetic field needs to be taken into account. The conservation equations and the Maxwell's equations are solved by the PC based software PHOENICS [4], which is sufficiently powerful for the simulation of the operation of auto-expansion and rotary/self-pressurizing circuit breakers. The behavior of electric arc burning in pure $\mathrm{SF}_{6}$ and in pure $\mathrm{N}_{2}$ is investigated in a high voltage autoexpansion circuit breaker.

\section{THE ARC MODEL}

Since the flow in a supersonic nozzle is axis-symmetric, the governing equations that describe the arc behaviours in the nozzle can be written in cylindrical polar coordinates $(r, z)$ system:

$$
\frac{\partial(\rho \varphi)}{\partial t}+\nabla \bullet(\rho \varphi \vec{V})-\nabla \bullet\left(\Gamma_{\varphi} \nabla \varphi\right)=S_{\varphi}
$$

where $\varphi, \Gamma_{\varphi}$ and $S_{\varphi}$ are, respectively, the dependent variable, the diffusion coefficient, and the source terms, which are listed in Table 1 for the mass, momentum, and energy equations, where all notations have their conventional meaning [5]. The subscript $l$ denotes the laminar part of the diffusion coefficient and $t$ the turbulent part. $q$ in the energy equation represents the net radiation loss per unit volume and time. It is calculated with the approximate model of Zhang et al [6]. Equation (1) assumes local thermodynamic equilibrium. Thus, the thermal and transport properties 
are functions of pressure and

TABLE I

SOURCE TERMS AND DIFFUSION COEFFICIENTS FOR GOVERNING EQUATIONS

\begin{tabular}{|lccc|}
\hline \multicolumn{1}{|c}{ Equation } & $\varphi$ & $\Gamma_{\varphi}$ & $\mathrm{S}_{\varphi}$ \\
\hline Continuity & 1 & 0 & 0 \\
\hline $\begin{array}{l}\text { Momentum } \\
\text { in } z \text { direction }\end{array}$ & $w$ & $\mu_{l}+\mu_{t}$ & $-\partial P / \partial z+$ viscous terms \\
\hline $\begin{array}{l}\text { Momentum } \\
\text { in } r \text { direction }\end{array}$ & $v$ & $\mu_{l}+\mu_{t}$ & $-\partial P / \partial r+$ viscous terms \\
\hline $\begin{array}{l}\text { Energy } \\
\text { (Enthalpy) }\end{array}$ & $h$ & $\left(k_{l}+k_{t}\right) / c_{p}$ & $\sigma E^{2}-q+d P / d t+$ viscous dissipation \\
\hline
\end{tabular}

temperature, which are tabulated and put into the program. The properties of $\mathrm{SF}_{6}$ from Murphy [7], and $\mathrm{N}_{2}$ from Yos [8] are used. The gases are assumed to be ideal at temperatures below $500 \mathrm{~K}$. Lorentz force and Ohmic heating resulting from the arcing current are included in the source terms of the momentum and energy equations, respectively. The current density and the electric field are calculated by solving the current continuity equation, which is expressed in terms of electrical potential:

$$
\nabla \bullet(\sigma \nabla \phi)=0
$$

where $\sigma$ is electrical conductivity and $\phi$ is the electrical potential. The Lorentz force is computed based on the axial and radial current densities and the azimuthal component of the magnetic field induced by the arcing current. Turbulenceenhanced momentum and energy transport are modelled by the Prandtl mixing length model. The turbulent viscosity is calculated by:

$$
\mu_{t}=\rho l_{m}^{2}\left|\frac{\partial w}{\partial r}\right|
$$

where $\rho$ is gas density and $l_{m}$ is the length scale. $l_{m}$, in the arc region is taken as a fraction of the arc thermal radius defined as the radial distance from the axis to the position of $4,000 \mathrm{~K}$ isotherm.

\section{RESULTS AND DISCUSSION}

The governing equations and the current continuity equation are solved by a commercial CFD package called PHOENICS [4]. The geometry of the $245 \mathrm{kV}$ auto-expansion circuit breaker under investigation is schematically shown in Fig. 1. The circuit breaker is initially filled at an absolute pressure of 0.69 MPa at room temperature for both cases of filling with gases of $\mathrm{SF}_{6}$ and $\mathrm{N}_{2}$. The electric field is of long range and requires a calculation domain bigger than the flow field, which is confined within the breaker. The domain is constructed by adding an annular block in the radial direction to the flow field domain based on the circuit breaker structure, which is schematically illustrated in Fig. 1. The radial dimension of this added area is varied until the calculated electric field in the circuit breaker is no longer sensitive to the radial boundary conditions of the extended domain.

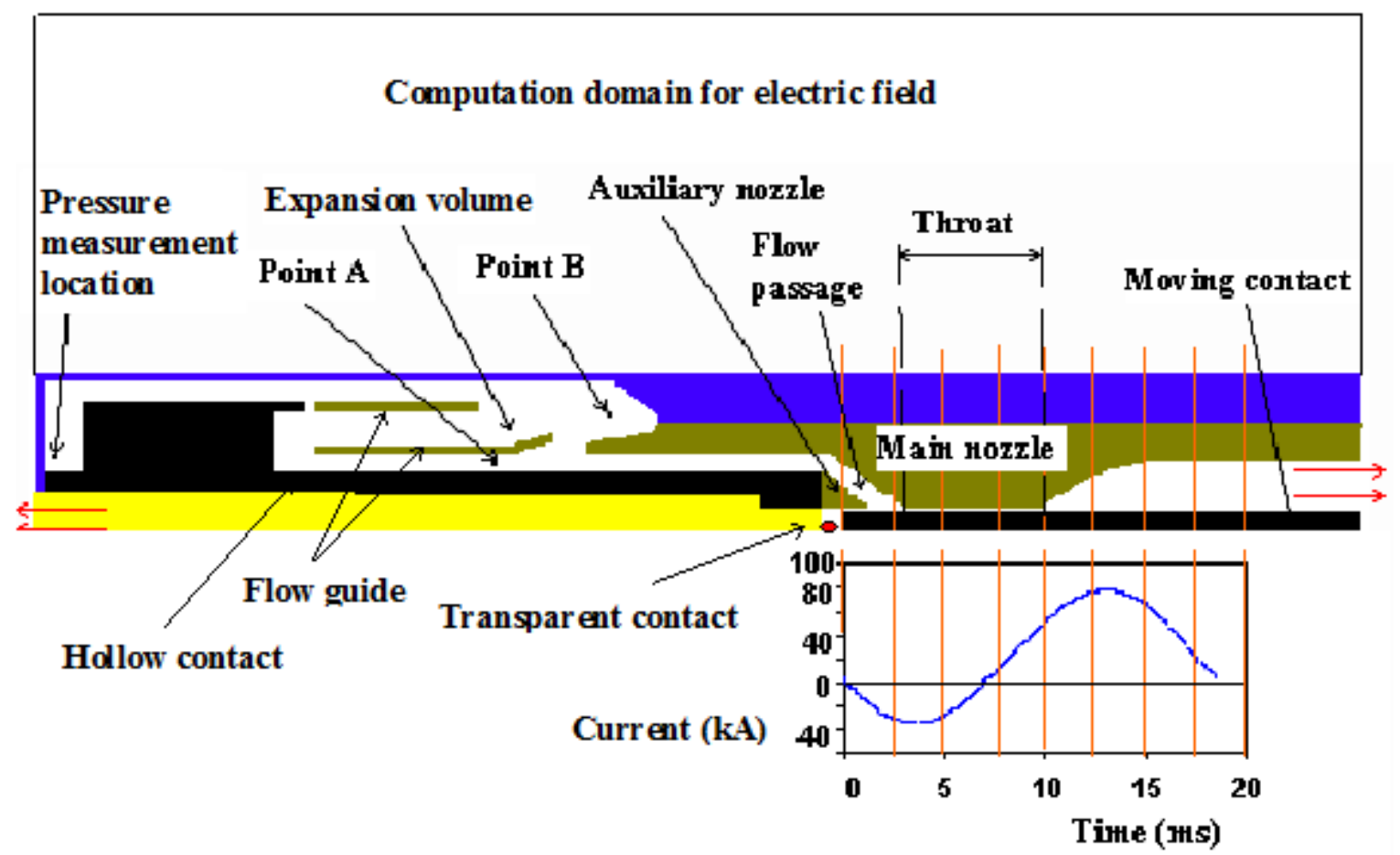

Fig. 1 Schematic diagram of the auto-expansion circuit breaker under investigation. The position of the moving solid contact and the instantaneous arcing current at different instants are also given. The region indicated as a transparent contact collects the current. It has no effect on gas flow inside the hollow contact. The vertical lines correspond to the positions of the moving contact at differe nt time. 
Within the flow field, body fitted coordinates (BFC) are used to model the complex geometry of the arcing chamber. Wall friction and energy loss by heat conduction at the nozzle and electrode surfaces are not taken into account because of their negligible effects on the arc behavior, especially at the high current period.

The physical phenomena of arc rooting inside the hollow

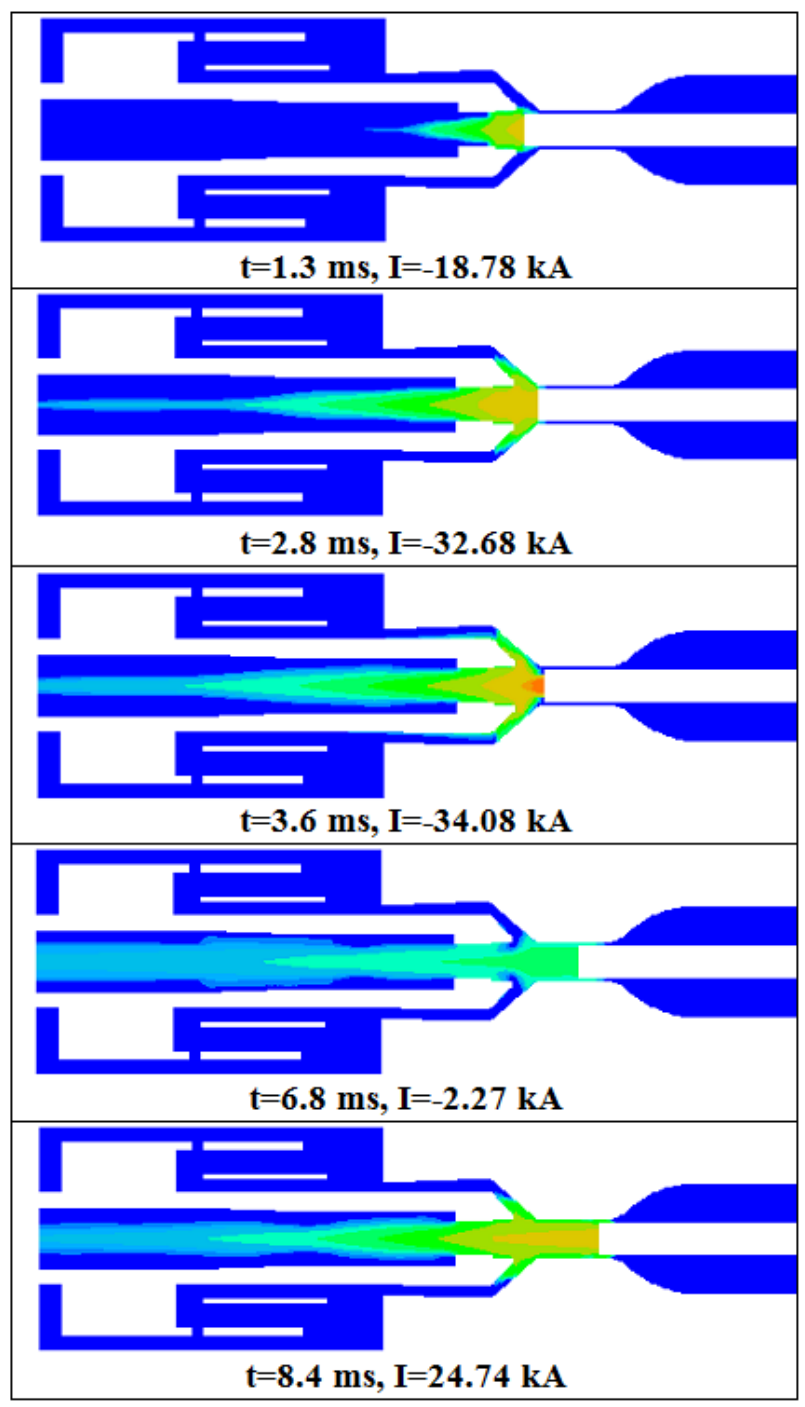

contact is extremely complex. The rooting mechanism is not exactly known. To simplify the three-dimensional problem of the arc root within the hollow contact, a transparent (to the flow) contact in the contact is placed and a circular spot is defined in the plane flush with the tip of the hollow contact to simulate the cathode spot. Its radius is calculated by assuming an average current density of $10^{8} \quad \mathrm{~A} / \mathrm{m}^{2}$.

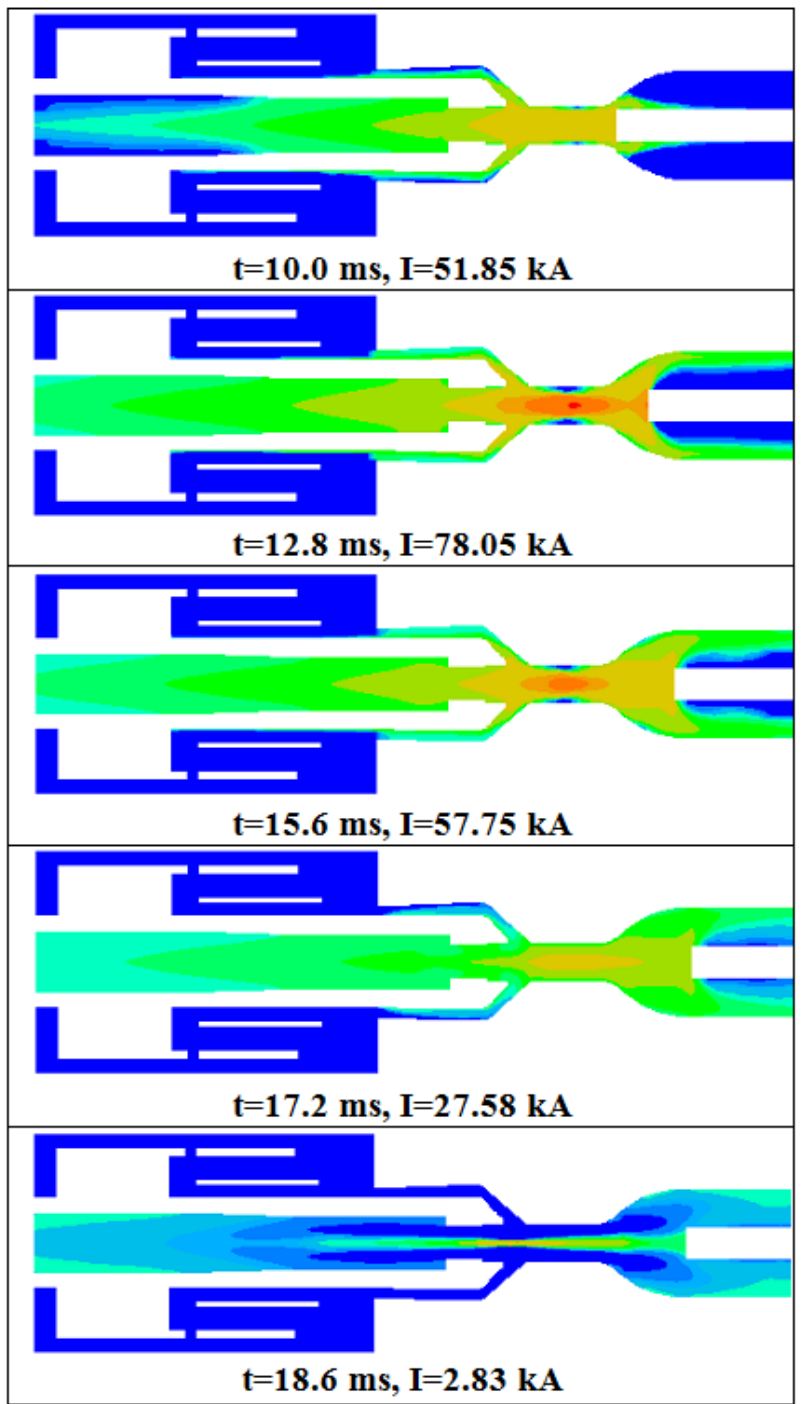

\section{Temperature scale (K)}

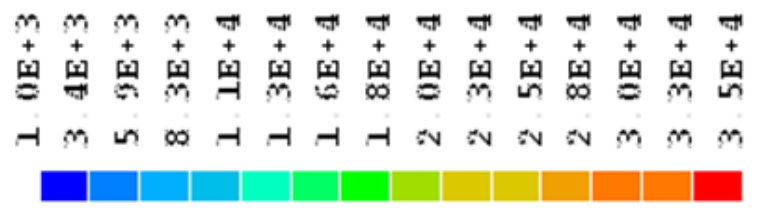

Fig. 2 Typical results of the temperature fields for the case of filling gas being $\mathrm{SF}_{6} . \mathrm{t}$ represents time, and I stands for the instantaneous current. 

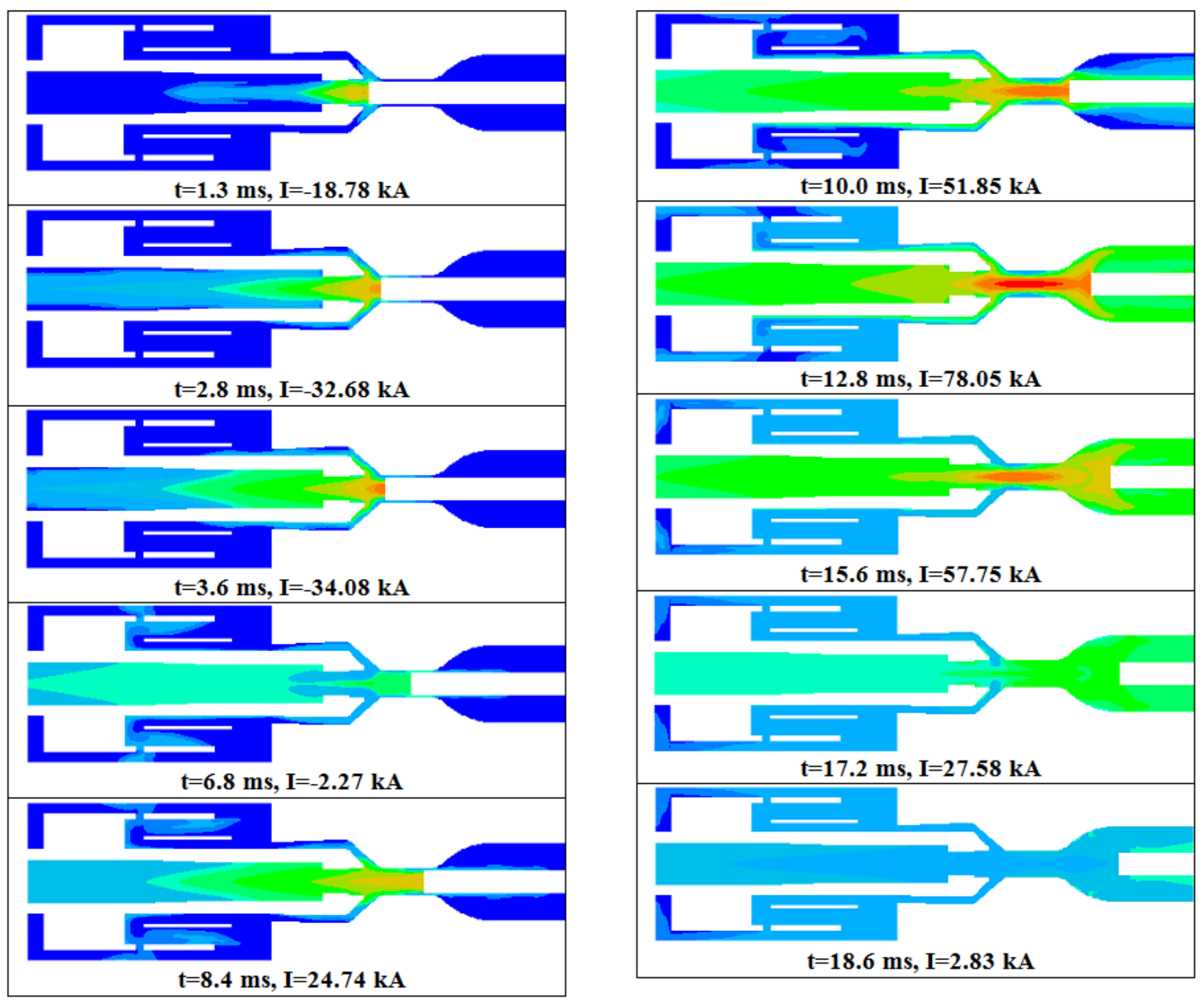

\section{Temperature scale (K)}

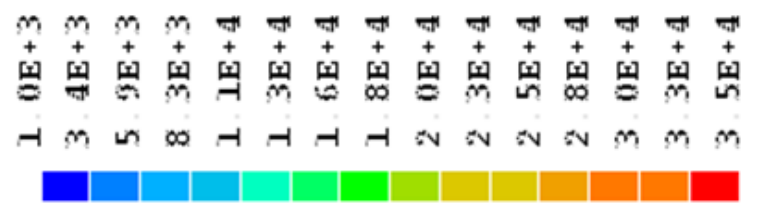

Fig. 3 Typical results of the temperature fields for the case of filling gas being $\mathrm{N}_{2}$. $\mathrm{t}$ represents time, and I stands for the instantaneous current.

The arc is initiated at a current of $2 \mathrm{kA}$ across a gap of 8 $\mathrm{mm}$ between the transparent and solid contacts by introducing a conducting plasma column across the gap. The column has a radius of $3 \mathrm{~mm}$, over which the gas temperature linearly decreases radially from $20,000 \mathrm{~K}$ at the centre to $300 \mathrm{~K}$ at the edge. The arcing duration is $18.6 \mathrm{~ms}$ with a second peak current of $78 \mathrm{kA}$ at $13 \mathrm{~ms}$ (Fig. 1).

The behaviour of electric arc burning in the mixture of $\mathrm{SF}_{6}$ and polytetrafluoroethylene (PTFE) vapour has been investigated in the $245 \mathrm{kV}$ auto-expansion circuit breaker (Fig.1) for the same current waveform shown in the same diagram [7]. The predicted arc voltage and pressure variation in the expansion volume are compared with the test results and they matched well. The same breaker has been used to investigate the effect of the filling gases on the arc behaviour here. The aim of the project here is to investigate the arc behaviors with different arc quenching and insulating mediums, $\mathrm{SF}_{6}$ and $\mathrm{N}_{2}$, therefore, the ablation of PTFE nozzle 
is not counted. In fact, when the instantaneous current falls below $30 \mathrm{kA}$, the PTFE mass concentration reduces to less than $5 \%$ and the arc starts to burn in a filling gas-dominated environment. At time $18.6 \mathrm{~ms}$, when the instantaneous current is $2.8 \mathrm{kA}$, there is only $2 \%$ PTFE vapor by mass in the main nozzle [7]. Therefore, the thermal recovery of the circuit breaker immediately after current zero can be regarded as taking place in a pure filling gas environment.

Two filling gases, $\mathrm{SF}_{6}$ and $\mathrm{N}_{2}$, have been investigated. Figs. 2 and 3 show that the temperature fields at a few different moments with the filling gases being $\mathrm{SF}_{6}$ and $\mathrm{N}_{2}$ respectively.

Generally, during the high current period, the pictures of the temperature fields with the two fillings are similar, but when the current drops below $30 \mathrm{kA}$, the arc column could not shrink in radial direction properly with Nitrogen, especially when the current drops towards its final zero. This phenomenon also observed by the investigation on the arc behaviour in a simple supersonic nozzle [9]. By comparing the material properties of the two gases, the properties of Nitrogen under low temperature $(\leq 5000 \mathrm{~K})$ should be responsible for it. Further and more detail investigation of the effects of the material properties on the arc behaviour will be continuously studied.

\section{CONCLUSION}

The behavior of an electric arc burning in two filling materials, $\mathrm{SF}_{6}$ and $\mathrm{N}_{2}$, has been investigated in a $245 \mathrm{kV}$ auto-expansion circuit breaker. The results show that, with the circuit breaker geometry under investigation, the temperature fields with the two fillings are similar during the high current period. However, when the current drops below $30 \mathrm{kA}$, the arc column could not shrink in radial direction properly with Nitrogen, especially when the current drops towards its final zero. The properties of Nitrogen under low temperature $(\leq 5000 \mathrm{~K})$ should be responsible for this.

\section{REFERENCES}

[1] A. R. Ravishankara, S. Solomon, A. A. Turnipseed, and R. F. Warren, "Atmospheric lifetimes of long-lived halogenated species", Science, Vol. 259, No. 5092, PP. 194-199, 1993.

[2] Kynast, and K. Juhre, "N2 and N2-CO2 Mixture in Gas Insulated Compartments under High Pressure," in Proc. 10th International Symposium on Gaseous Dielectrics, Greece, 2004, pp. 18.

[3] T. Uchii, Y. Hoshina, T. Mori, H. Kawano, T. Nakamoto, and H. Mizoguchi, "Investigations on SF6-free Gas Circuit Breaker Adopting $\mathrm{CO} 2$ Gas as an Alternative Arc Quenching and Insulating Medium," in Proc. 10th International Symposium on Gaseous Dielectrics, Greece, 2004, pp. 17.

http://dx.doi.org/10.1007/978-1-4419-8979-6_28

[4] CHAM, Phoenics, London, U.K.

[5] J. D. Yan, M. T. C. Fang, and W. Hall, "The development of PC based CAD tools for auto-expansion circuit breaker design", IEEE Trans. Power Delivery, Vol. 14, PP. 176-181, Jan. 1999.

[6] J. F. Zhang, M. T. C. Fang, and D. B. Newland, "Theoretical Investigation of a 2KA DC Nitrogen Arc in a Supersonic Nozzle", J. Phys. D, Appl. Phys., Vol. 20, PP. 368-379, 1987.
[7] J. L. Zhang, J. D. Yan, A. B. Murphy, W. Hall, and M. T. C. Fang, "Computational Investigation of Arc Behaviour in an Auto-Expansion Circuit Breaker Contaminated by Ablated Nozzle Vapour", IEEE Trans. Plasma Science, Vol. 30, PP. 706-719, 2002.

[8] J. M. Yos, Report, Liverpool University, UK.

[9] J. L. Zhang, "Computational Investigation of Effects of $\mathrm{SF}_{6}, \mathrm{CO}_{2}$ and $\mathrm{N}_{2}$ on Arc Interruption in a Supersonic Nozzle," in Proc. 20th International Conference on Gas discharges and Their Applications (GD2014), Orleans, France, 2014, pp. 95-98.

Jinling Zhang was born in Shaanxi, China. She received the B.Sc., M.Sc., and $\mathrm{Ph} . \mathrm{D}$. degrees from the Department of Energy and Power Engineering, Xi'an Jiaotong University, Xi'an, China, in 1982, 1987, and 1995, respectively.

She was a Lecturer at Xi'an Jiaotong University between 1987 and 1996, and then became an Associate Professor before she joined the Department of Electrical Engineering and Electronics, the University of Liverpool, U.K., in 1997 as a Research Fellow working on circuit-breaker arcs. She is a Lecturer in the Department of Electrical Engineering and Electronics, Xi'an JiaotongLiverpool University with research interests in thermal plasma and computational fluid dynamics. 